\title{
Breathing Air into Clinical Care
}

\author{
Molly Scannell Bryan ${ }^{a, c}$ Robert Sargis ${ }^{b, c, d}$ \\ aDepartment of Medicine, Institute for Minority Health Research, University of Illinois at Chicago, Chicago, IL, \\ USA; ${ }^{b}$ Department of Medicine, University of Illinois at Chicago, Chicago, IL, USA; ${ }^{c}$ Chicago Center for Health and \\ Environment, Chicago, IL, USA; dJesse Brown Veterans Affairs Medical Center, Chicago, IL, USA
}

In their current article entitled "Ambient Air Pollution and Mortality among Older Patients Initiating Maintenance Dialysis," Feng and colleagues [1] have added to an emerging tapestry of data that links fine particulate matter air pollution $\left(\mathrm{PM}_{2.5}\right)$ to adverse kidney outcomes. Using patient information from the United States Renal Data System (USRDS), the authors analyzed data from almost 400,000 individuals aged 65 years or older initiating dialysis, and found higher death rates if the patient resided in zip codes where average annual $\mathrm{PM}_{2.5}$ levels exceeded $12 \mu \mathrm{g} / \mathrm{m}^{3}$, while the risk began at lower levels among those older than 75 years. The authors' findings of increased mortality associated with $\mathrm{PM}_{2.5}$ highlights how exposure to poor air quality may have life-threatening consequences, particularly among those who are medically complex, including older patients with ESRD. While there remains additional work to be done in this area, notably individual-ascertainment of exposures as well as consideration of the various constituents of air pollution, the article's conclusions complement ample animal, ecological, and epidemiologic evidence that all suggest that air quality influences the development, progression, and severity of kidney disease [2-4]. Furthermore, the impact of air pollution on kidney disease is now recognized as a

karger@karger.com

(C) 2021 S. Karger AG, Basel

www.karger.com/ajn

Karger! global problem of urgent concern in various regions of the world [4].

We welcome the attention that Feng et al.'s [1] research draws to the associations between poor air quality and kidney disease. With this attention, we now emphasize an underappreciated corollary of their findings, namely, that in order to appropriately treat their patients, clinicians must advocate for individual- and populationlevel interventions that reduce their patients' exposure to poor air quality.

We write this editorial in recognition of a near-universal neglect of environmental exposures as critical, and crucially, modifiable determinants of kidney health. Studies such as Feng and colleagues' [1] provide abundant reason for those who treat patients across the spectrum of kidney disease to be concerned about the deleterious impact of air pollution. We must do better.

We recognize that the ecological nature of air quality undoubtedly creates challenges for how to best incorporate exposure mitigation into the practice of medicine; however, Feng et al. [1] suggest that failing to address those risk factors can be a matter of life or death. The question only becomes how do we move from our knowledge of the importance of good air quality to actionable 
changes to clinical practice that will support patient wellness.

We advocate for a two-pronged approach to such an initiative. First, among clinicians who treat kidney disease, we support engaging with patients about the importance of healthy air across their disease course. We can advise patients to avoid outdoor activities when and where air pollution is high, and we can encourage alternatives to practices that worsen local air quality, [5] such as refraining from smoking or vaping indoors; refraining from the residential burning of wood, leaves, or trash; replacing health-disrupting household chemicals; choosing electric rather than gas-powered utilities and tools; and the installation of indoor high-efficiency particulate air (HEPA) filters. In recognition of their power to raise awareness within the nephrology community, we also see a special role for professional organizations, in that they can educate clinicians about how improvements to air quality have the potential to augment patients' health in ways that rival many of the individual-level interventions that are standard components of patient care.

However, while individually tailored advice is what clinicians are trained to provide, in this context, such advice can create the impression that individual actions are sufficient. Such narrow guidance would exaggerate a patient's agency in controlling their exposure to poor air quality. As such, we emphasize the need for a second prong to the approach that is grounded in a clear awareness that the genesis of much air pollution is external to the home, [6] and therefore exposure is largely invariant to individual choices. As such, we are calling on clinical providers and their professional organizations to advocate in support of policies that improve air quality in order to better care for our patients. We make this recommendation through the lens of health justice, as communities that are disproportionately impacted by kidney disease are also those that are exposed to higher levels of air pollution [7, 8]. Indeed, the findings of Feng et al. [1] demonstrate these disparities, as older Black patients in their study had a higher $\mathrm{PM}_{2.5}$-associated mortality risk than non-Hispanic whites. While these systematically disadvantaged communities are bearing the burden of policy choices that expose them to poor air quality, it is imperative for those who treat them to advocate for the policy changes that could rectify this disparity.

As part of this effort, we ask for professional organizations to bring air quality and other environmental exposures to the attention of their societies' members through the drafting of professional practice guidelines that highlight poor air quality as a nephrotoxic risk factor. Currently, there is an unfortunate and gaping chasm between environmental health knowledge and clinical practice guidelines. Indeed, each of the prominent guidelines for kidney disease clinical practice, the Kidney Disease Outcomes Quality Initiative, the Kidney Disease Improving Global Outcomes, and the Veterans Affairs/Department of Defense Clinical Practice Guidelines, is largely silent on the role of environmental pollution in the pathogenesis and progression of kidney disease. At the least, revisions to these guidelines should highlight the need for future research and strengthen the ability of the nephrology community to address this underappreciated risk factor. Moreover, revised guidelines should support a comprehensive recognition of the role of environmental health in the development and clinical consequences of chronic diseases, which in turn would help de-politicize environmental policy among our community and refocus the discussion on improving human health. Indeed, this is a critical opportunity for the nephrology community to lead broader efforts to improve clinical care since air pollution is a clinically neglected contributor linked to many chronic conditions [9].

Study after study has demonstrated that health and disease are influenced by the ecosystems in which people live. Air pollution has the potential to be one of the strongest of these social determinants of health that can be readily modified. As such, clinicians must engage with these social determinants of health as they endeavor to reduce the incidence and burden of chronic disease. Feng and colleagues [1] have brought attention to the important link between air pollution and the health of patients on dialysis. Nestled into the context of the broader literature, these data should trigger a salvo of acts by the medical community to both encourage individual action and, crucially, to advocate for strong environmental policy to prioritize policy interventions that will improve the health of all of our patients.

\section{Conflict of Interest Statement}

R.M.S. has received honoraria from CVS/Health and the American Medical Forum; neither of these impact this manuscript.

\section{Funding Sources}

M.S.B. is supported by the National Institutes of Health (OT2OD026557) and the Patient Centered Outcomes Research Network (CDRN-1306-04737). R.M.S. is supported by the National Institute of Environmental Health Sciences (R01 ES028879, R21 ES030884, and P30 ES027792). 


\section{References}

1 Feng Y, Jones MR, Chu NM, Segev DL, McAdams-DeMarco, M. Ambient air pollution and mortality among older patients initiating maintenance dialysis. Am J Nephrol. 2021, DOI:10.1159/000514233

2 Liu B, Fan D, Huang F. Relationship of chronic kidney disease with major air pollutants: a systematic review and meta-analysis of observational studies. Environ Toxicol Pharmacol. 2020;76:103355.

3 Al Suleimani YM, Al Mahruqi AS, Al Za'abi M, Shalaby A, Ashique M, NemmarA, et al. Effect of diesel exhaust particles on renal vascular responses in rats with chronic kidney disease. Environ Toxicol. 2017;32:541-9.
4 Bowe B, Xie Y, Li T, Yan Y, Xian H, Al-Aly Z. Particulate matter air pollution and the risk of incident CKD and progression to ESRD. J Am Soc Nephrol. 2018;29(1):218-30.

5 Sargis RM, Heindel JJ, Padmanabhan V. Interventions to address environmental metabolism-disrupting chemicals: changing the narrative to empower action to restore metabolic health. Front Endocrinol. 2019;10:33.

6 Bernstein JA, Alexis N, Bacchus H, Bernstein IL, Fritz P, Horner E, et al. The health effects of non-industrial indoor air pollution. J Allergy Clin Immunol. 2008;121(3):585-91.
7 Ruiz D, Becerra M, Jagai JS, Ard K, Sargis RM. Disparities in environmental exposures to endocrine-disrupting chemicals and diabetes risk in vulnerable populations. Diabetes Care. 2018;41(1):193-205.

8 Mikati I, Benson AF, Luben TJ, Sacks JD, Richmond-Bryant J. Disparities in distribution of particulate matter emission sources by race and poverty status. Am J Public Health. 2018;108(4):480-5.

9 Brook RD, Newby DE, Rajagopalan S. Air pollution and cardiometabolic disease: an update and call for clinical trials. Am J Hypertens. 2017;31:1-10. 